\title{
Neutrino factory near detector
}

\author{
M. Bogomilov, Y. Karadzhov, R. Matev, ${ }^{*}$ and R. Tsenov \\ Department of Atomic Physics, St. Kliment Ohridski University of Sofia, Sofia, Bulgaria
}

A. Laing and F. J. P. Soler

University of Glasgow, Glasgow, United Kingdom

(Received 31 December 2012; published 7 August 2013)

\begin{abstract}
The neutrino factory is a facility for future precision studies of neutrino oscillations. A so-called near detector is essential for reaching the required precision for a neutrino oscillation analysis. The main task of the near detector is to measure the flux of the neutrino beam. Such a high intensity neutrino source like a neutrino factory provides also the opportunity for precision studies of various neutrino interaction processes in the near detector. We discuss the design concepts of such a detector. Results of simulations of a high resolution scintillating fiber tracker show that such a detector is capable of determining the neutrino flux normalization with an uncertainty of less than $1 \%$ by measuring pure leptonic interactions. Reconstruction of the neutrino energy in each event and a flux estimation based on the shapes of the neutrino energy spectra are discussed. A full setup of the near detector, consisting of a high granularity vertex detector, high resolution tracker, and muon catcher is also presented. Finally, a method to extrapolate the measured near detector flux to the far detector is shown, demonstrating that it is able to extract the correct values of $\theta_{13}$ and the $C P$ violation phase $\delta$ without any significant bias and with high accuracy.
\end{abstract}

DOI: $10.1103 /$ PhysRevSTAB.16.081001

PACS numbers: 07.77.Ka, 29.40.Mc, 13.15.+g, 14.60.Pq

\section{NEUTRINO FACTORY NEAR DETECTOR(S) BASELINE}

A future neutrino facility, such as a neutrino factory (NF) [1], will need near detectors in order to perform oscillation measurements with the required sensitivity. As a neutrino factory simultaneously accelerates $\mu^{+}$and $\mu^{-}$beams, it is necessary to have one near detector at each of the straight sections of the storage ring, thus at each of the two polarities (see Fig. 1). The near detector tasks include measurement of neutrino flux through the measurement of neutrino-electron scattering, measurement of neutrino beam properties needed for the flux to be extrapolated to the far detector, and measurement of charm production cross sections (charm production in the far detector is one of the principal backgrounds to the oscillation signal). In addition, the high intensity neutrino factory beam enables unique neutrinonucleus scattering physics studies, such as the measurement of cross sections, structure functions, nuclear effects, $\sin ^{2} \theta_{W}$, etc. The near detector must also be capable of searching for new physics, for example by detecting $\tau$ leptons, which are particularly sensitive probes of nonstandard interactions at source and at detection, and since $\nu_{\tau}$ detection is also important in the search for sterile neutrinos.

\footnotetext{
*Corresponding author. rmatev@phys.uni-sofia.bg

Published by the American Physical Society under the terms of the Creative Commons Attribution 3.0 License. Further distribution of this work must maintain attribution to the author(s) and the published article's title, journal citation, and DOI.
}

Design requirements for the near detector(s) can be formulated as follows: a low $\mathrm{Z}$ high resolution tracker for flux and cross-section measurement $\left(\nu_{\mu}\right.$ and $\left.\nu_{e}\right)$; a magnetic field for improved muon momentum resolution compared to the far detector [the magnetized iron neutrino detector (MIND)] [2]; with a muon catcher for muon identification; capability for $\mathrm{e}^{+} / \mathrm{e}^{-}$identification; a vertex detector for charmed hadron and $\tau$-lepton detection (for nonstandard interactions and sterile neutrinos searches); and good neutrino energy resolution (better than in the far detector) for flux extrapolation.

The current near detector design anticipates three subdetectors (Fig. 2): a high granularity detector for charm $/ \tau$ measurement; a high resolution tracker for precise measurement of the event close to the vertex; and a mini-MIND detector for muon measurement. The paper will describe

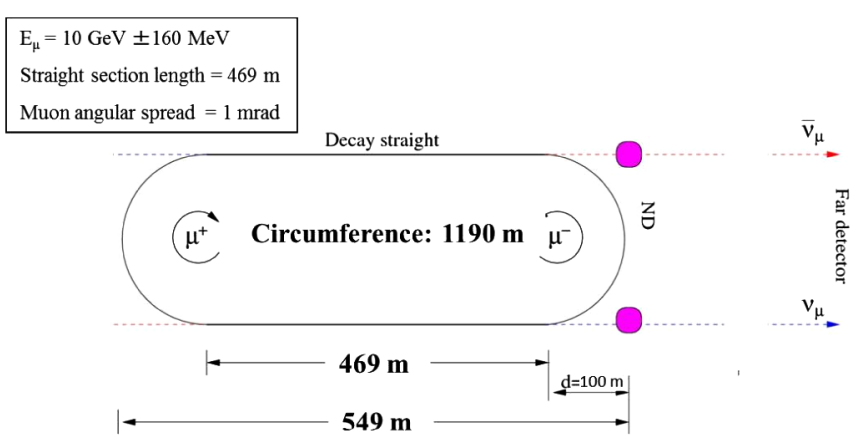

FIG. 1. Baseline locations of the near detectors. The decay ring parameters are given for a $10 \mathrm{GeV}$ neutrino factory. 


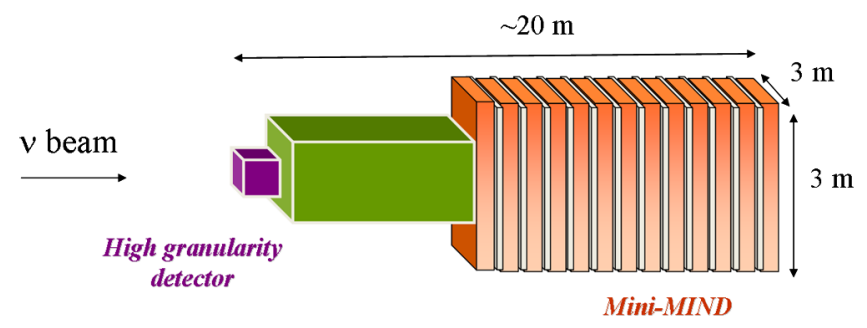

FIG. 2. Block diagram design of the near detector.

the design concept and simulation of a high resolution tracker based on scintillating fibers. An alternative design of the tracker is considered in parallel in [1], namely the HiResM $\nu$ detector [3], where tracking is performed by planes of straw tubes.

The paper will commence with a description of how the near detector can perform a measurement of neutrino flux by neutrino-electron scattering (Sec. II), it then proceeds to describe the scintillating fiber tracker (Sec. III), the scintillating fiber simulation (Sec. IV), the reconstruction of the lepton candidates in the scintillating fiber tracker (Sec. V), the leptonic event selection (Sec. VI), background subtraction (Sec. VII), neutrino energy reconstruction (Sec. VIII), and the determination of the neutrino flux energy distribution (Sec. IX). The final two sections include a description of the high granularity charm and tau detector (Sec. X) and a method for extrapolating from the near to the far detector in the neutrino oscillation analysis at a neutrino factory (Sec. XI) before a final conclusion.

\section{MEASUREMENT OF THE NEUTRINO FLUX BY NEUTRINO-ELECTRON SCATTERING}

Neutrino-electron interaction cross sections are straightforward to calculate in the standard model [4]. Any small uncertainties arise only from (well measured) standard model parameters. Therefore, such processes are suitable for measurement of neutrino beam fluxes, provided that beams are intense enough.

There are two pure leptonic neutrino interactions that produce an energetic muon in the final state:

$\nu_{\mu}+\mathrm{e}^{-} \rightarrow \mu^{-}+\nu_{\mathrm{e}}$ and $\bar{\nu}_{\mathrm{e}}+\mathrm{e}^{-} \rightarrow \mu^{-}+\bar{\nu}_{\mu}$ (IMD).

The first one is known as inverse muon decay (IMD), while the second one produces a muon in the final state through annihilation. The neutrino energy $\left(E_{\nu}\right)$ threshold (for electrons at rest) for both processes is $10.9 \mathrm{GeV}$.

There are four pure leptonic neutrino reactions of interest, which are referred to as elastic scattering (ES), producing an energetic electron:

$$
\begin{gathered}
\nu_{\mu}+\mathrm{e}^{-} \rightarrow \nu_{\mu}+\mathrm{e}^{-} \text {and } \bar{\nu}_{\mathrm{e}}+\mathrm{e}^{-} \rightarrow \bar{\nu}_{\mathrm{e}}+\mathrm{e}^{-}\left(\mathrm{ES}^{-}\right) \\
\nu_{\mathrm{e}}+\mathrm{e}^{-} \rightarrow \nu_{\mathrm{e}}+\mathrm{e}^{-} \text {and } \bar{\nu}_{\mu}+\mathrm{e}^{-} \rightarrow \bar{\nu}_{\mu}+\mathrm{e}^{-}\left(\mathrm{ES}^{+}\right) .
\end{gathered}
$$

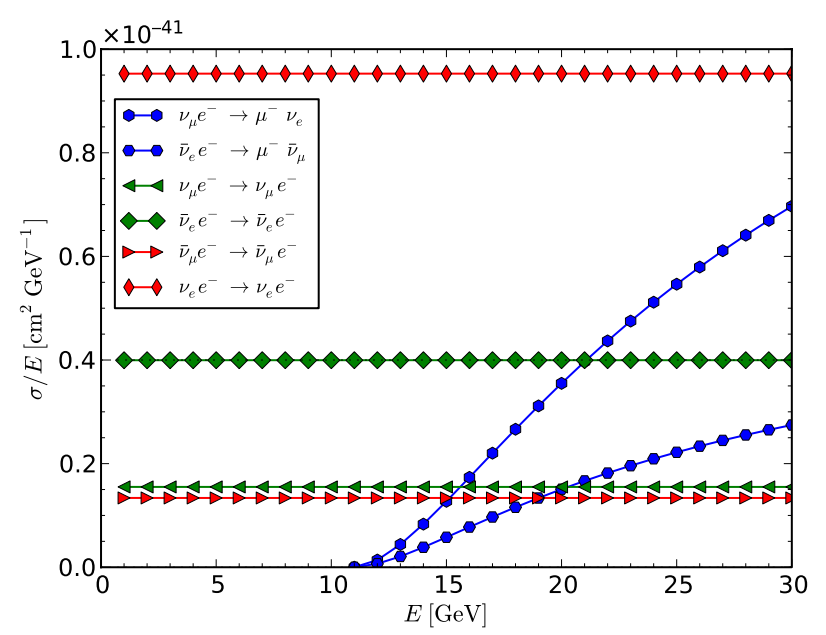

FIG. 3. Total cross section divided by neutrino energy for the leptonic interactions in a neutrino factory beam. Hexagon markers show $\mathrm{CC}$ interactions, triangle markers show $\mathrm{NC}$ interactions, and rhomboid markers show mixed $\mathrm{CC}+\mathrm{NC}$ interactions. The threshold for quasielastic processes (1) (blue) is evident at $\sim 11 \mathrm{GeV}$.

The processes (2) will appear in a $\mu^{-}$decay mode beam, while the processes (3) are relevant for a $\mu^{+}$decay mode beam. In what follows we shall often refer to the processes (1)-(3) as leptonic processes/events.

The total cross sections for the above processes as a function of the neutrino energy are shown in Fig. 3. Despite their smallness, a massive detector placed close to the straight section of the neutrino factory storage ring can provide sufficient interaction rate; see Fig. 4 for the case of a neutrino factory with $25 \mathrm{GeV}$ muon energy.

However, inclusive charged-current (CC) and neutralcurrent (NC) neutrino interactions with nuclei

$$
\nu_{\ell}+N \rightarrow \ell+X \quad \text { and } \quad \nu_{\ell}+N \rightarrow \nu_{\ell}+X
$$

have cross sections a few orders of magnitude larger. An obvious distinction between purely leptonic processes and the processes in (4) is the lack of a hadronic system $X$ in the former. Thus, the measured recoil energy of the hadronic system can be used as a good criterion for background suppression. Muons from the quasielastic process (1) have an angular distribution peaked in the very forward direction. At a $25 \mathrm{GeV}$ neutrino factory, the polar angle $\left(\theta_{l}\right)$ of these muons does not exceed $5 \mathrm{mrad}$. The angular spread comes mainly from the intrinsic scattering angle $\sim 4 \mathrm{mrad}$ in these processes, while the neutrino beam divergence and solid angle covered by the detector make little contribution. This kinematic property can be used as another event selection criterion. The polar angle distribution of electrons from neutrino-electron elastic scattering (2) and (3) is 10 times wider and is not suitable for event selection. On the other hand, the composite variable $\theta_{\ell}^{2} E_{\ell}$, proportional to the Bjorken variable $y=1-E_{l} / E_{\nu}$ in elastic scattering, where $E_{l}$ is the lepton energy, provides good 


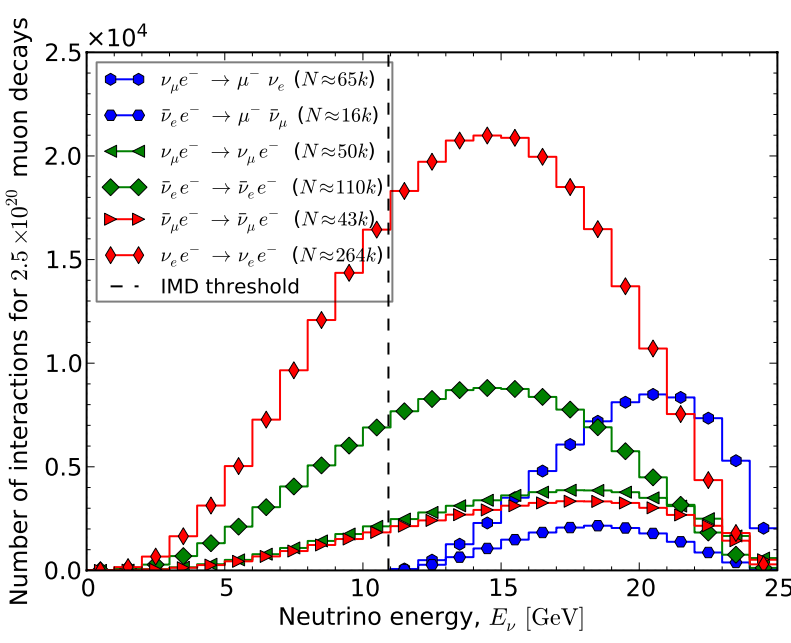

FIG. 4. Number of neutrino-electron interactions for a nominal year of neutrino factory operation $\left(2.5 \times 10^{20}\right.$ muon decays per muon charge per straight section). Rates are calculated for a $2.7 \mathrm{t}$ detector with $1.5 \times 1.5 \mathrm{~m}^{2}$ frontal cross section and average $Z / A \approx 0.54$. The detector is placed $100 \mathrm{~m}$ after the straight section of the neutrino factory with $25 \mathrm{GeV}$ muon energy. The dashed vertical line indicates the threshold for quasielastic scattering.

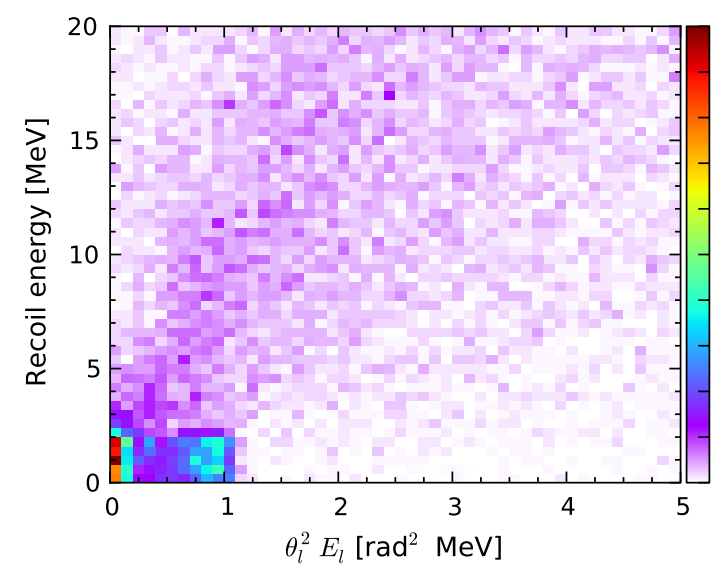

FIG. 5. True $\theta_{\ell}^{2} E_{\ell}$ vs true recoil energy plus deposited energy in $1 \mathrm{~cm}$ of polystyrene by the scattered lepton. The peak at $\theta_{\ell}^{2} E_{\ell} \sim 0$ (1) $\operatorname{rad}^{2} \mathrm{MeV}$ corresponds to IMD (ES $\left.{ }^{-}\right)$events. A $25 \mathrm{GeV} \mu^{-}$beam is considered. separation between signal and background for all neutrinoelectron scattering processes, provided the lepton angle and energy are measured with sufficient precision. The discrimination power of the $\theta_{\ell}^{2} E_{\ell}$ and recoil energy of the hadronic system are shown in Fig. 5.

\section{SCINTILLATING FIBER TRACKER}

A schematic drawing of a scintillating fiber tracker with an incorporated calorimeter is shown in Fig. 6. The detector consists of 20 square shaped modules placed perpendicular to the beam axis. Each module has a calorimeter section and a tracker section (also called tracker station). Modules are placed in equidistant positions, forming gaps filled with air. With a larger distance between tracker stations, the $X$ and $Y$ displacement of hits is increased and thus the angular resolution is improved. The sides of the air gaps are covered with layers of plastic scintillation bars. The detector is placed in a $0.5 \mathrm{~T}$ dipole magnetic field. Each station consists of one layer of fibers with horizontal orientation and another one with vertical orientation. Each layer has four planes made of $1 \mathrm{~mm}$ cylindrical fibers. They form a hexagonal pattern in the layer, thus minimizing the dead volume. There are 12000 fibers per station, thus 240000 fibers in total. The calorimeter sections consist of plastic scintillation bars perpendicular to the magnetic field and arranged in five planes in each section. Bars are coextruded with a wavelength shifting (WLS) fiber inside and have $10 \mathrm{~mm} \times 30 \mathrm{~mm}$ cross section. Both the tracker fibers and WLS fibers in the bars are read from both ends by silicon photomultipliers (SiPMs). Overall dimensions of the detector are $\sim 1.5 \mathrm{~m} \times 1.5 \mathrm{~m} \times 11 \mathrm{~m}$ and the detector mass is $\sim 2.7 \mathrm{t}$.

\section{SIMULATION OF THE DETECTOR RESPONSE}

The neutrino flux at the near detector was generated by a Monte Carlo simulation of muon decays along the straight section of the neutrino factory decay ring $[5,6]$. Neutrino interactions in the detector were simulated by the GENIE package [7]. For the simulation of the detector response, the GEANT4 software platform [8] was used.

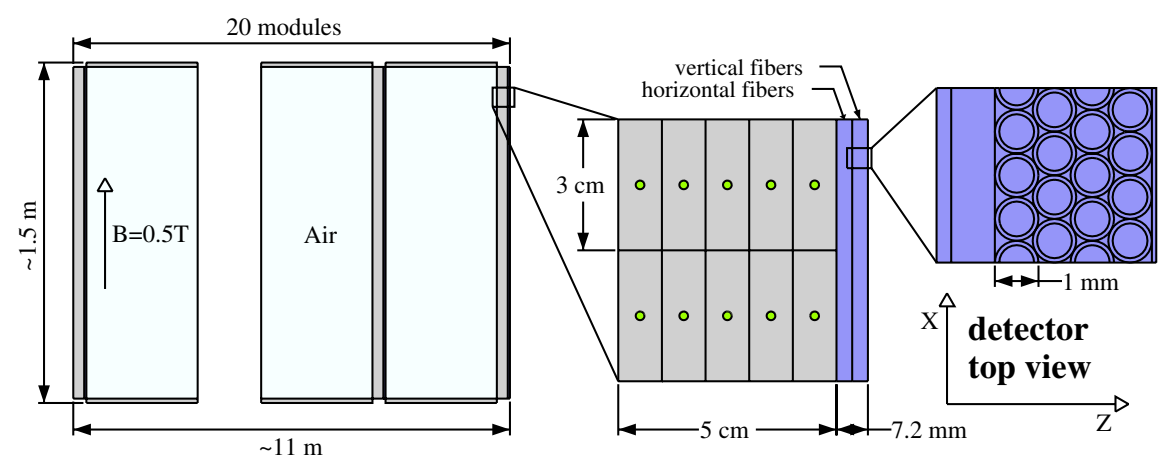

FIG. 6. Schematic drawing of the scintillating fiber tracker. 
The presence of a muon catcher (e.g. miniature version of MIND) as a subdetector is essential for the muon identification. For various reasons we have not included it in the simulation. Instead, we assume that a muon having momentum of at least $500 \mathrm{MeV}$ is identified in the near detector with $100 \%$ efficiency. Such an assumption is not unreasonable since a muon with momentum of $500 \mathrm{MeV}$ has a range of $\approx 170 \mathrm{~cm}$ in polystyrene, thus it will leave the tracker and enter the catcher, where it will be measured.

Precise and efficient momentum determination of electrons by measuring their track curvature is not possible in the rather dense polystyrene tracker due to bremsstrahlung and partial electromagnetic (EM) shower development. Therefore, electron energy should be measured by calorimetric means. The scintillating fiber tracker is an excellent totally active electromagnetic calorimeter, but not all the energy is captured in it due to its rather large radiation length. This implies the need for a dense electromagnetic calorimeter surrounding the scintillating fiber tracker. We assume that in such an enclosing detector almost all of the electromagnetic energy is captured and measured with a resolution of $6 \% / \sqrt{\mathrm{E} / \mathrm{GeV}}$ [9]. In the current simulation, if the electromagnetic energy deposition [as estimated from the Monte Carlo (MC)] of an event without a muon is less than $500 \mathrm{MeV}$, the event is discarded.

\section{Digitization}

A simple Monte Carlo algorithm was developed to simulate the processes of scintillation, photon propagation inside the fibers, and SiPM response. For each charged particle hit in a fiber, a random number of scintillation photons is drawn according to the energy deposition and scintillation yield of the material. The fraction of photons remaining in the fiber (trapping efficiency) and light attenuation are taken into account. The photons from multiple hits are summed up and an optical coupling efficiency factor is applied to get the number of incident photons on the SiPM surface. Multiplying the latter by the photon detection efficiency, one obtains the number of primary avalanche triggers in the SiPMs. Dark counts are simulated by further adding a Poisson distributed number to the primary triggers. The final electronic signal response is parametrized by a function that takes into account cross-talk effect and the single pixel response distribution. Only signals with amplitude larger than a threshold equivalent to 2.5 fired pixels in each of the photodetectors at the ends of the fibers are retained.

It seems that the dark counts are not a major problem for $1 \mathrm{~mm}$ fibers and for modern SiPMs, which have less than $1 \mathrm{MHz} / \mathrm{mm}^{2}$ dark count rate at room temperature. With the imposed signal threshold and required coincidence of the signals from both ends of a fiber, the number of "false" fiber digits is below $1 \%$ of all the fibers in the detector. Therefore, the probability to have two or more adjacent false fiber digits which would mimic true particle hits is negligible.

The bar signals are digitized by smearing energy deposits with a $20 \%$ Gaussian. Signals below a threshold equivalent to a deposited energy of $0.5 \mathrm{MeV}$ are discarded.

\section{RECONSTRUCTION OF THE LEPTON CANDIDATE}

First, the neighboring fired fibers are grouped into clusters. Depending on the fiber orientation, the $(x, z)$ or $(y, z)$ position of the cluster is calculated by taking the weighted average of the fiber positions. All clusters consistent with a single particle traversing the layer at small angle (with respect to the $z$ axis) are marked.

To measure the angle of the scattered lepton track, only events with one cluster per orientation in the first two stations are considered. This selection has low efficiency, because an event with a single charged particle in the final state might still have more than one cluster in either of the first stations. (For example, consider $\delta$ electrons or interactions of bremsstrahlung photons.) Using measured points from subsequent stations does not improve the angle measurement precision. This is due to the fact that the absorber thickness, the module spacing, and the fiber size are chosen so that the rms deviation due to multiple scattering is of the same order or larger than the hit resolution.

For events with a muon (identified in the muon catcher), the muon track is reconstructed by the following procedure. First, only clusters which are alone in their layer and are marked as consistent with a single particle energy deposition are selected. Then, a track seed is created by fitting a parabola to the measurement points defined from the clusters. Further on, the RECPACK package [10] is used to apply a Kalman filter [11-13] fitting procedure to the measurements starting from the computed seed. Finally, the fired fibers from the unused stations are assigned to the track according to a distance requirement and the track is refitted. Unfortunately, this sophisticated fitting procedure did not improve the track momentum resolution compared to the seed and it even deteriorated the precision of the initial angle measurement. Those observations might indicate for an issue with the RECPACK software package that seemingly fails to take into account multiple scattering and energy loss correctly. We have not investigated further this fitting procedure for the following reasons. Only marginal resolution improvement on the initial angle is expected from using all track hits as opposed to using only the first two (see the paragraph above). Second, for the purpose of measuring neutrino flux via leptonic events, the uncertainty in the scattering angle dominates both the uncertainty of the 

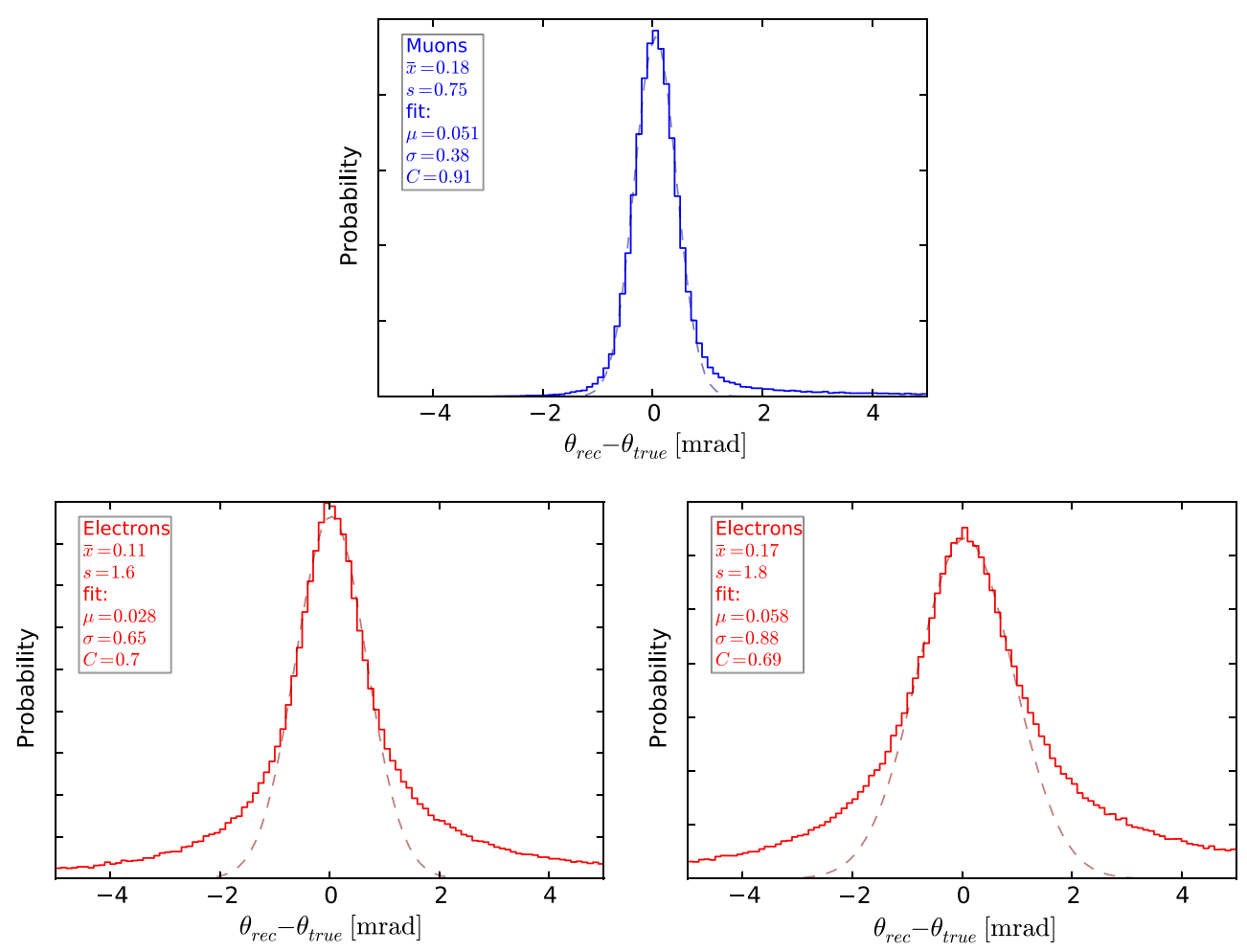

FIG. 7. Detector angular resolution for muons (top) and electrons (bottom left: $25 \mathrm{GeV}$; bottom right: $10 \mathrm{GeV}$ neutrino factory) for leptonic events. Gaussian fits are shown with dashed lines. The sample mean, standard deviation, and fit parameters are shown in the upper left corners.
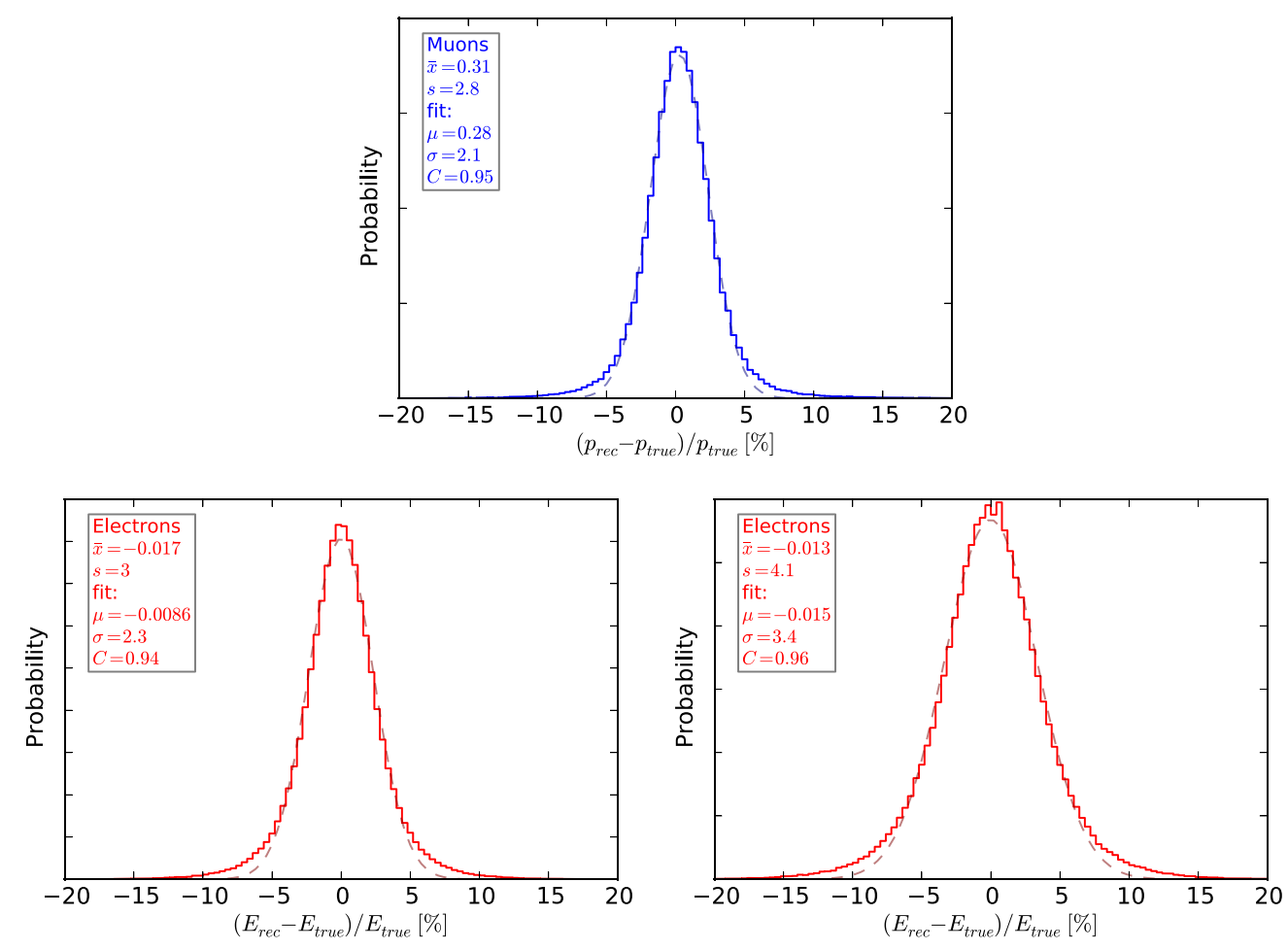

FIG. 8. Detector momentum resolution for muons (top) and electrons (bottom left: $25 \mathrm{GeV}$; bottom right: $10 \mathrm{GeV}$ neutrino factory) for leptonic events. Gaussian fits are shown with dashed lines. The sample mean, standard deviation, and fit parameters are shown in the upper left corners. 
main selection variable $\theta_{\ell}^{2} E_{\ell}$ and the reconstructed neutrino energy uncertainty. In the following we use the value from the parabolic fit for the muon momentum.

For events with no muon, Monte Carlo information for the neutrino interaction is used to get the total electromagnetic energy deposition. We iterate over the final state particles of the neutrino reaction and sum the energies of all $e^{ \pm}, \gamma$, and $\pi^{0}$. The summed up energy is then smeared with the assumed resolution $(6 \% / \sqrt{\mathrm{E} / \mathrm{GeV}})$. Since in the following analysis we are interested in events with a single lepton in the final state, the value obtained this way is assumed to be the energy of the electron candidate track.

The fraction of leptonic events remaining after the above reconstruction procedure is $61 \%$ for IMD and $52 \%$ for $\mathrm{ES}$ for a $25 \mathrm{GeV}$ neutrino factory, and $43 \%$ for ES for a $10 \mathrm{GeV}$ scenario.

As a result of the reconstruction procedure, for the scattered lepton track we obtain the initial position, initial slopes $\left(x_{0}^{\prime}=d x / d z\right.$ and $\left.y_{0}^{\prime}=d y / d z\right)$, initial momentum, and charge.

Distributions of the differences between reconstructed and true value of the lepton scattering angle and momentum for both the 25 and $10 \mathrm{GeV}$ neutrino factory scenarios are shown in Figs. 7 and 8. The asymmetric behavior for the muons is mostly due to the fact that $\theta_{l}$ is a positively defined polar angle, constructed from the measured angles in the $X Z$ and $Y Z$ planes. The non-Gaussian tails seen for electrons can be attributed to events with early bremsstrahlung.

\section{SELECTION OF LEPTONIC EVENTS}

At a $25 \mathrm{GeV}$ neutrino factory three event samples can be selected: IMD and $\mathrm{ES}^{-}$samples in the $\mu^{-}$decay mode and $\mathrm{ES}^{+}$sample in the $\mu^{+}$decay mode. In a $10 \mathrm{GeV}$ scenario the neutrino energy is below the IMD threshold, thus only ES samples remain. For the latter scenario we impose a veto on events with a muon identified in the muon catcher.

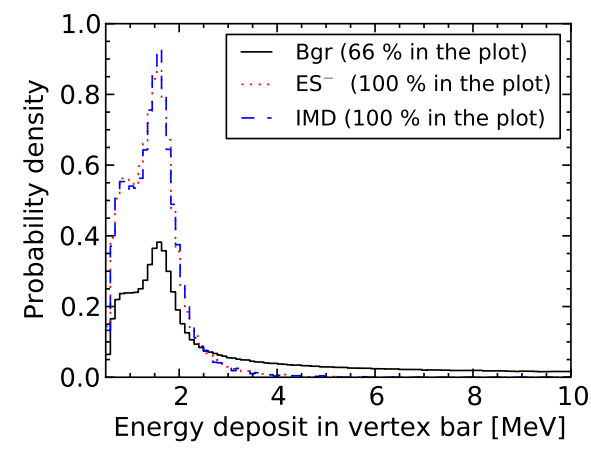

\section{A. Calorimetric selections around the vertex}

Both IMD and ES events have a property of low (consistent with single particle) energy deposition near the vertex. To exploit that, a cut on energy deposition in the vertex bar of $4 \mathrm{MeV}$ is imposed. If the vertex is in a cluster of fibers, a cut is made on the sum of fiber amplitudes. In some background events, energetic charged hadrons (hundreds of $\mathrm{MeV}$ ) escape through the air gaps leaving small or no depositions in the calorimetric sections. Therefore, it is required that there is no activity in the side bars covering the air gaps adjacent to the vertex. Another vertex related cut is the requirement that there are no energy depositions upstream of the vertex (backward depositions). The distribution of the energy deposition in the vertex bar is shown in Fig. 9 (left).

\section{B. Other calorimetric selections}

To select IMD events, one can rely on the specific properties of the muon energy loss $(d E / d x)$. At energies in the range of $11-25 \mathrm{GeV}$, a muon is nearly a minimum ionizing particle. Therefore, the following cuts were applied when selecting IMD events: the mean of the depositions in all slabs is less than $3 \mathrm{MeV}$ and the maximum deposition is less than $12 \mathrm{MeV}$. For ES events, the scattered electron could induce a shower in the detector, thus such cuts are not suitable. In this case a variable characterizing the transverse spread of the fired bars relative to the lepton track, $x_{\mathrm{dev}}$, is constructed. A selection cut on $x_{\mathrm{dev}}$ exploits the spatial symmetry of energy depositions relative to the track in the nonbending plane for events with a single scattered particle. For selecting IMD events, the $x_{\mathrm{dev}}$ variable cut is at $2.5 \mathrm{~cm}$, while for ES events a more relaxed cut at $15 \mathrm{~cm}$ is applied. The distribution of the $x_{\mathrm{dev}}$ variable is shown in Fig. 9 (right).

\section{Kinematic selections in case of $25 \mathrm{GeV} \boldsymbol{\mu}^{-}$beam}

As the signal events always have a negatively charged lepton $\left(\mu^{-}\right.$or $\left.\mathrm{e}^{-}\right)$, a cut $q / p<0$ on the scattered track is imposed. Quasielastic neutrino-electron scattering has a

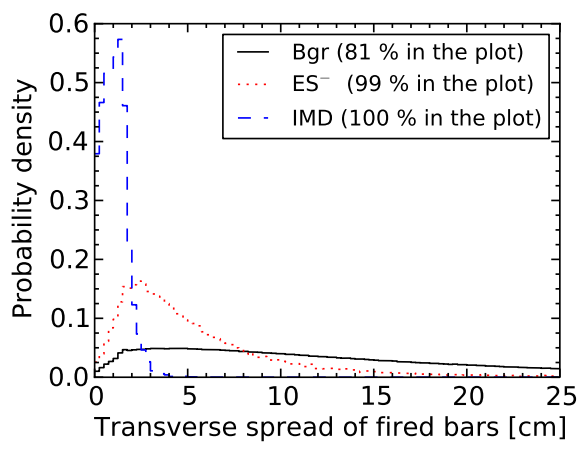

FIG. 9. Distributions of the energy deposited in the vertex bar (left) and $x_{\mathrm{dev}}$ variable (right) for IMD (blue), ES ${ }^{-}$(red), and background (black) events in $25 \mathrm{GeV} \mu^{-}$mode. The fraction of events contained in the plot is indicated in the legend. All distributions are normalized to a unit area. This plot shows only events for which the reconstructed vertex is in a scintillator bar. 

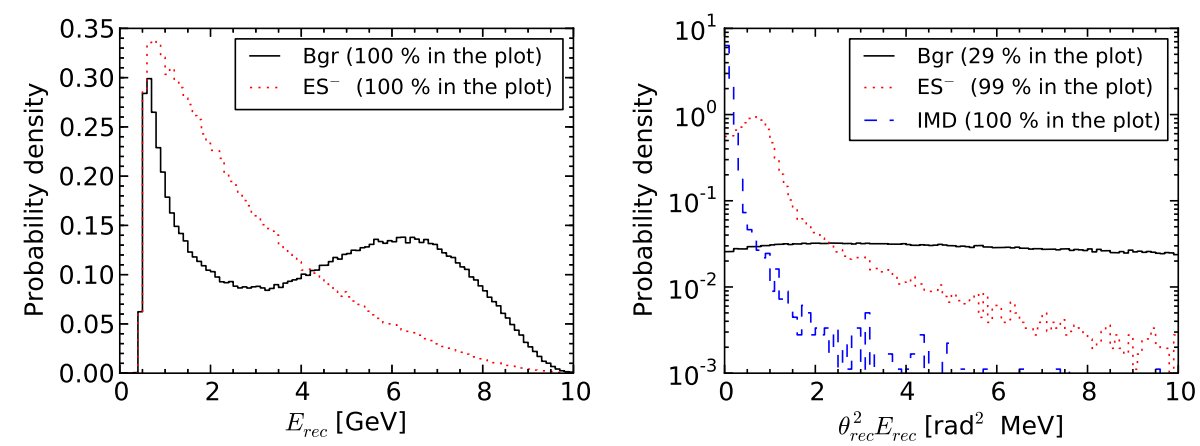

FIG. 10. Left: Distribution of measured total electromagnetic energy for $\mathrm{ES}^{-}$(red) and background (black) events in the $\mu^{-}$ decay mode of a $10 \mathrm{GeV}$ neutrino factory. Right: Distribution of the reconstructed $\theta_{\ell}^{2} E_{\ell}$ variable for the scattered track for IMD (blue), $\mathrm{ES}^{-}$(red), and background (black) events in the $\mu^{-}$decay mode of a $25 \mathrm{GeV}$ neutrino factory. The fraction of events contained in the plot is indicated in the legend. All distributions are normalized to a unit area.

threshold at $\sim 11 \mathrm{GeV}$. Therefore, when selecting the IMD sample, events with scattered track momenta of less than $10 \mathrm{GeV}$ are discarded. In order to avoid contamination of IMD events in the $\mathrm{ES}^{-}$sample, only events with scattered track momentum less than $10 \mathrm{GeV}$ are retained in this sample [14]. This is a reasonable cut, since the majority of the $\mathrm{ES}^{-}$events have scattered track momentum of less than $10 \mathrm{GeV}$. The overall efficiencies of the selection cuts are $89 \%$ for the IMD sample, $73 \%$ for the $\mathrm{ES}^{-}$sample, and $87 \%$ for the $\mathrm{ES}^{+}$sample.

\section{Kinematic selections in case of $10 \mathrm{GeV}$ neutrino factory}

Here, in addition to the negative charged track cut, we impose a cut on the measured total electromagnetic energy. There is a significant difference in shape of the total EM energy distributions for leptonic and background events, see Fig. 10 (left). This enables us to reject events with a total EM energy of more than $5 \mathrm{GeV}$. With such a cut, the background is reduced by a factor of $\approx 3$, while reducing the signal selection efficiency by $\approx 20 \%$.

\section{BACKGROUND SUBTRACTION}

It is evident that an absolutely clean sample of signal events cannot be selected with a reasonable efficiency by employing selection cuts only. Therefore, the estimation of certain background distributions and their extrapolation to the signal region should be made, with the aim of statistically subtracting the background in the selected event samples.

We have chosen to do background subtraction in terms of the scattered lepton ( $\mu$ or e) angle and momentum, measured in the detector. They provide the most powerful signal-to-background separation due to the kinematic properties of the signal processes (1)-(3).

For simplicity, we perform a one-dimensional analysis. In the case of the IMD signal extraction, the scattering angle $\theta_{\ell}$ and composite variable $\theta_{\mu}^{2} E_{\mu}$ can be used to discriminate the signal from the background. In the case of the ES signal, the background is well separated only when one exploits $\theta_{\mathrm{e}}^{2} E_{\mathrm{e}}$. The distributions of $\theta_{\ell}^{2} E_{\ell}$ for the IMD signal, the $\mathrm{ES}^{-}$signal, and the background for a $25 \mathrm{GeV}$ scenario are shown in Fig. 10 (right). It is seen that the background distribution over the $\theta_{\ell}^{2} E_{\ell}$ variable is nearly flat. This fact allows for a simple parametrization of the background distribution. The subsequent analysis is made using only the $\theta_{\ell}^{2} E_{\ell}$ variable.

Methods for obtaining the number of signal events are discussed below.

\section{A. Linear fit method}

The linear fit method relies on the nearly flat shape of the corresponding background distribution. The idea is to estimate the background under the signal peak by linear extrapolation from the signal-free region. First, an interval over the $\theta_{\ell}^{2} E_{\ell}$ distribution is defined so that its lower limit is close to the signal peak, there are almost no signal events in the interval (according to the MC simulation) and the background is approximately linear in this interval. Then, the histogram is fitted with a straight line in the interval. Finally, the line is extrapolated towards zero to estimate the number of background events under the signal peak. The histograms over $\theta_{\ell}^{2} E_{\ell}$ and the linear fits for the event samples under consideration are shown in Fig. 11. Comparison between the estimated and the true number of signal events is given in Table I [15]. It is seen that the true values lie within the $95 \%$ confidence intervals of the predictions.

The main source of systematic errors related to the method is the assumption about the linearity of the background shape. Signal and background events are obtained with the Monte Carlo neutrino interaction generator we use (GENIE). The background in this very specific part of the phase space cannot be considered well understood and implemented in any of the available Monte Carlo 

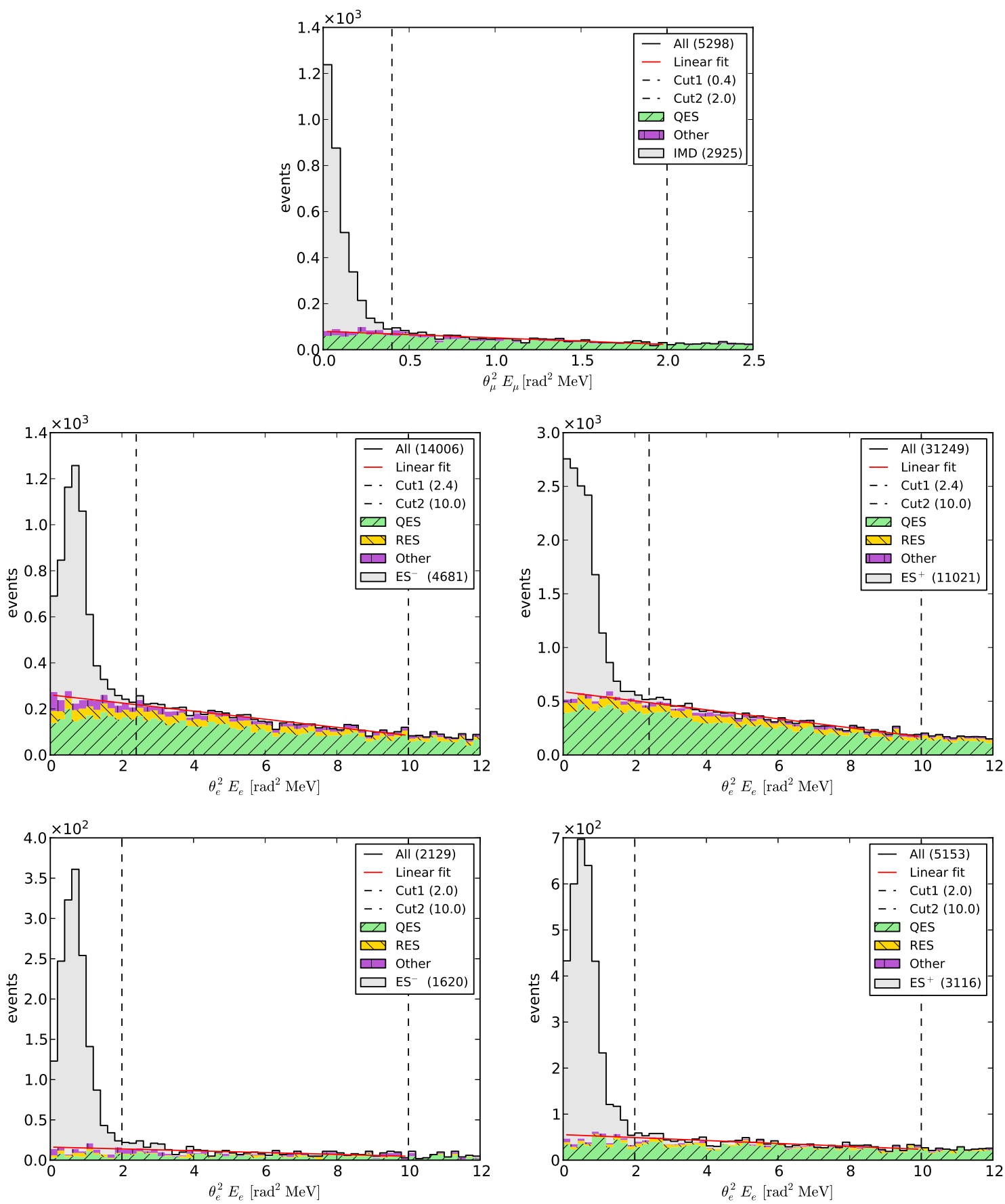

FIG. 11. Distributions over $\theta_{\ell}^{2} E_{\ell}$ for the different event samples considered. In each histogram the distribution of the leptonic events is filled with solid grey, the distribution of the hadronic events is hatched, and the total spectrum is the black histogram. The two cuts bounding the fit interval are drawn with dashed lines. The red line indicates the background extrapolation. The top three histograms refer to the $25 \mathrm{GeV}$ scenario: IMD sample (top), $\mathrm{ES}^{-}$sample (middle left), and $\mathrm{ES}^{+}$sample (middle right), while the bottom two are for the $10 \mathrm{GeV}$ neutrino factory: $\mathrm{ES}^{-}$sample on the bottom left and $\mathrm{ES}^{+}$sample on the bottom right.

generators. In view of this we choose the linear model as the simplest one. From the fit of the $\mathrm{ES}^{-}$and $\mathrm{ES}^{+}$samples it is estimated that a systematic error of less than $1 \%$ can be achieved. However, to give a conclusive estimation, one should investigate if and how various parameters of the simulation and selections influence the background shape.

\section{B. $\mu^{+}$method}

IMD interactions are present only in the $\mu^{-}$decay mode. The idea of the $\mu^{+}$method is to estimate the background under the IMD signal peak exploiting the distribution of positive muons detected in the neutrino beam $\left(\bar{\nu}_{\mu}, \nu_{\mathrm{e}}\right)$ originated from $\mu^{+}$decays. This method 
TABLE I. Estimated number of signal events for the three event samples. For the $25 \mathrm{GeV}(10 \mathrm{GeV})$ scenario, the statistics correspond to $2.3 \times 10^{19}\left(5 \times 10^{19}\right) \mu^{-}$decays and as many $\mu^{+}$decays, which is $9.2 \%(10 \%)$ of a nominal year.

\begin{tabular}{|c|c|c|c|c|c|c|c|}
\hline Event sample & Selection efficiency & Overall efficiency & Purity & All events & Signal events & Signal events linear fit & $\mu^{+}$method \\
\hline \multicolumn{8}{|c|}{$25 \mathrm{GeV}$ muon beam } \\
\hline IMD & $86 \%$ & $46 \%$ & $81 \%$ & 3520 & 2850 & $2926 \pm 59$ & $2831 \pm 61$ \\
\hline $\mathrm{ES}^{-}$ & $70 \%$ & $32 \%$ & $61 \%$ & 7355 & 4491 & $4479 \pm 86$ & \\
\hline $\mathrm{ES}^{+}$ & $83 \%$ & $37 \%$ & $63 \%$ & 16964 & 10607 & $10512 \pm 131$ & \\
\hline \multicolumn{8}{|c|}{$10 \mathrm{GeV}$ muon beam } \\
\hline $\mathrm{ES}^{-}$ & $68 \%$ & $29 \%$ & $92 \%$ & 1637 & 1511 & $1486 \pm 40$ & \\
\hline $\mathrm{ES}^{+}$ & $64 \%$ & $28 \%$ & $86 \%$ & 3414 & 2951 & $2892 \pm 58$ & \\
\hline
\end{tabular}

was previously used for measuring of the IMD process in the CHARM-II detector $[16,17]$. In the near detector, an event sample from the $\left(\bar{\nu}_{\mu}, \nu_{\mathrm{e}}\right)$-beam events is selected with the same selection cuts as for the IMD sample. For consistency, we again consider the $\theta_{\mu}^{2} E_{\mu}$ distribution. To account for the difference in the $\nu_{\mu} N$ and $\bar{\nu}_{\mu} N$ event rates, the $\theta_{\mu}^{2} E_{\mu}$ histogram for the $\mu^{+}$should be normalized to the $\theta_{\mu}^{2} E_{\mu}$ histogram for the $\mu^{-}$. First, the ratio of the $\mu^{-}$background histogram and the $\mu^{+}$histogram is calculated, see Fig. 12 (right). It is seen, that the ratio under the signal peak $\left(\theta_{\mu}^{2} E_{\mu}<0.4 \operatorname{rad}^{2} \mathrm{MeV}\right)$ is at the same level as the ratio outside the signal peak. An interval outside the IMD signal peak and with approximately constant ratio of $\mu^{-}$and $\mu^{+}$events is chosen as $\Delta=$ $[0.4,2.0] \mathrm{rad}^{2} \mathrm{MeV}$ and the normalization factor is then calculated as

$$
R=\frac{N_{\mu^{-}}^{\Delta}}{N_{\mu^{+}}^{\Delta}}=0.210 \pm 006,
$$

where $N_{\mu^{-}}^{\Delta}\left(N_{\mu^{+}}^{\Delta}\right)$ is the number of events in the interval $\Delta$ in the $\mu^{-}\left(\mu^{+}\right)$histogram. The $\mu^{-}$histogram and the normalized $\mu^{+}$histogram are shown in Fig. 12 (left). Apart from normalization, there are second order differences between the $\mu^{-}$and $\mu^{+}$distributions [16,17], which are not taken into account. The normalized $\mu^{+}$ histogram is then subtracted from the $\mu^{-}$one and the result gives the number of signal events (see the last column of Table I).

\section{RECONSTRUCTION OF THE NEUTRINO ENERGY}

\section{A. Kinematics of $\nu e^{-} \rightarrow l \nu$ scattering}

The energy-momentum conservation leads to the following expression for the incoming neutrino energy in the laboratory frame for a neutrino-electron scattering process:

$$
E_{\nu}=\frac{2 E_{l} m_{e}-m_{l}^{2}-m_{e}^{2}}{2\left(m_{e}-E_{l}+p_{l} \cos \theta_{l}^{\prime}\right)},
$$

where $E_{\nu}$ is the incoming neutrino energy, $E_{l}$ and $p_{l}$ are the outgoing lepton energy and momentum, $\theta_{l}^{\prime}$ is the outgoing lepton angle with respect to the incoming neutrino direction, and $m_{e}$ and $m_{l}$ are the electron and outgoing lepton masses. In the near detector the angle of the outgoing lepton with the $z$ axis $\theta_{l}$ is measured, rather than the angle $\theta_{l}^{\prime}$, see Fig. 13. Let the coordinate system be as shown in Fig. 13. The azimuthal angle of the neutrino can be
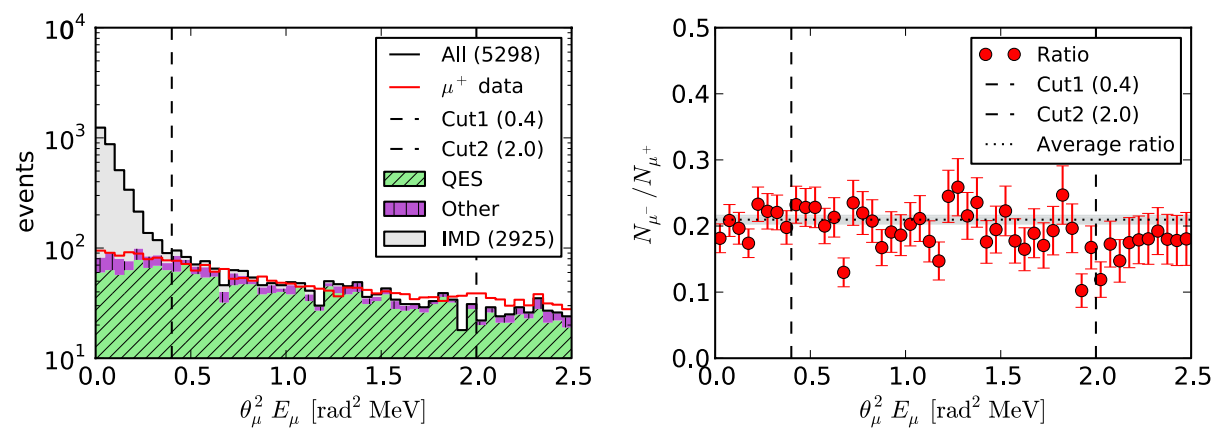

FIG. 12. The left plot shows distributions over $\theta_{\mu}^{2} E_{\mu}$ for the $\mu^{-}$beam sample. The leptonic events histogram is filled with solid gray, the hadronic events histogram is hatched, and the total spectrum is in black. The two cuts bounding the normalization region $\Delta$ are drawn with a dashed line. The red line indicates the normalized $\mu^{+}$histogram. The right plot shows the ratio of the $\mu^{-}$histogram and the $\mu^{+}$histogram over $\theta_{\mu}^{2} E_{\mu}$. The errors are statistical and the bars correspond to $1 \sigma$. The horizontal dotted line indicates the normalization ratio. 


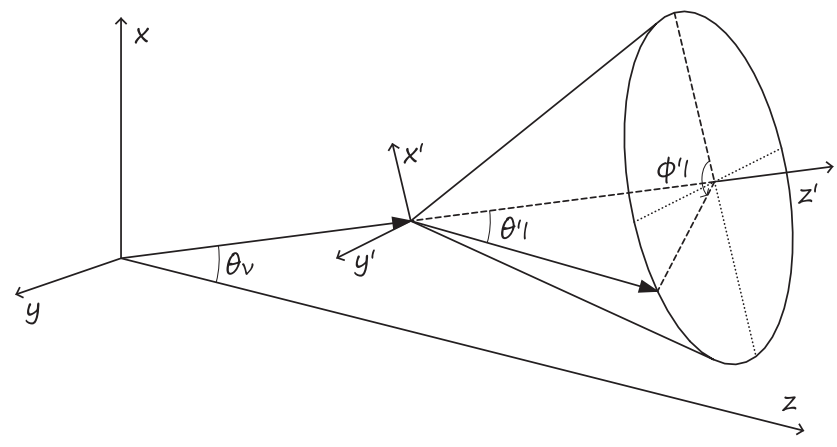

FIG. 13. Reference frame of the near detector (and the neutrino beam) $x y z$ and frame of the neutrino-electron scattering $x^{\prime} y^{\prime} z^{\prime}$ ( $z^{\prime}$ is along with the neutrino momentum).

determined by the position of the vertex in the detector. For simplicity, let us assume that it is zero. Then, the relation between $\theta_{l}^{\prime}$ and $\theta_{l}$ is given by

$$
\cos \theta_{l}^{\prime}=\cos \theta_{\nu} \cos \theta_{l}+\sin \theta_{\nu} \sin \theta_{l} \cos \varphi_{l},
$$

where $\theta_{\nu}$ is the neutrino angle with respect to the $z$ axis and $\varphi_{l}$ is the azimuthal angle of the lepton (in the $x y z$ frame).

\section{B. Naive neutrino energy reconstruction}

Let us use Eq. (6) with a simple assumption that $\theta_{l}^{\prime}=\theta_{l}$ to obtain the reconstructed neutrino energy $E_{\nu}^{\text {rec }}$. Such an assumption is well justified when the neutrino source length is small compared to the distance to the detector. Unfortunately, this is not the case here. To demonstrate the problem we take the lepton energy and angle with respect to the $z$ axis from the simulation (i.e. no detector resolution is involved). The migration matrix ( $E_{\nu}^{\text {rec }}$ vs $\left.E_{\nu}^{\text {true }}\right)$ and the relative difference $\left(E_{\nu}^{\text {rec }}-E_{\nu}^{\text {true }}\right) / E_{\nu}^{\text {true }}$ for the IMD event sample are shown in Fig. 14. It is seen that the difference has a broad distribution with a sample standard deviation of $23.5 \%$.

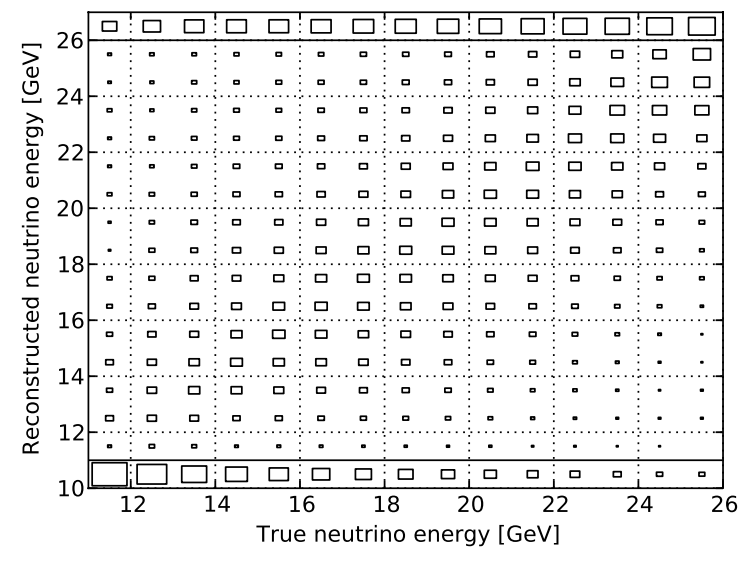

\section{Improved neutrino energy reconstruction}

The unknown angle $\theta_{\nu}$ can be constrained from the radial position of the vertex $r_{\mathrm{vtx}}$, the possible muon decay positions, and the $E_{\nu}<E_{\text {beam }}$ limit. Then one can average on the possible $\theta_{\nu}$ angles and estimate $\theta_{l}^{\prime}$ by using Eq. (7). A generalization of that method for measurement of neutrino energy on an event-by-event basis can be constructed by using a likelihood function $f(\vec{\eta} \mid \vec{x})$, where $\vec{\eta}$ represents unmeasurable (e.g. $E_{\nu}$ ) variables, $\vec{x}$ stands for quantities measured with the detector (e.g. $E_{l}$ ) and the value of the function is the event probability. The likelihood function should include as much information as possible: detector resolutions, kinematics, differential cross sections, and beam properties. In our case, we have constructed a likelihood function using the kinematic relations, the differential cross sections, and the true neutrino flux shapes (assuming a fair knowledge will be available from the beam instrumentation and Monte Carlo). The estimate for the neutrino energy is then obtained from

$$
E_{\nu}^{\text {meas }}=\int E_{\nu}(\vec{\eta}) f(\vec{\eta} \mid \vec{x}) d \vec{\eta}
$$

where $\vec{x}=\left(E_{l}, \theta_{l}, \varphi_{l}, r_{\mathrm{vtx}}\right)$. Significant improvement is achieved with this method. As it is seen in Fig. 15, the standard deviation for the IMD sample is reduced to $8.7 \%$. For the $10 \mathrm{GeV}$ neutrino factory scenario the best resolution for the reconstructed energy can be as good as 13\%, see Fig. 15, middle and bottom row of plots. Nevertheless, the ambiguity coming from the unknown neutrino angle (or muon decay position) cannot be fully eliminated on an event-by-event basis.

Migration matrices and resolutions for the reconstructed neutrino energy $E_{\nu}^{\text {meas }}$ in the $10 \mathrm{GeV}$ scenario obtained by using quantities measured in the detector are shown in Fig. 16. The neutrino energy resolution is now typically around $30 \%$.

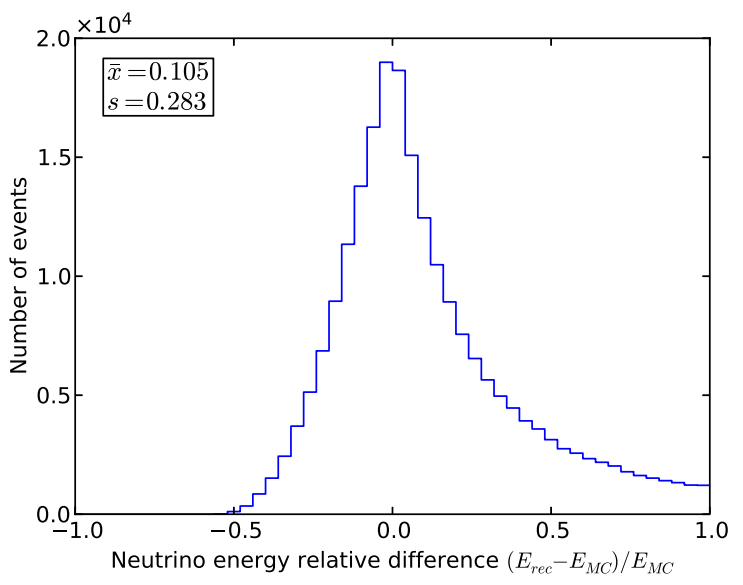

FIG. 14. Naive reconstruction of the neutrino energy using the IMD sample (background excluded). Left-migration matrix (the sum over each column is normalized to unity). The overflow and underflow bins are separated with horizontal lines. Rightrelative difference $\left(E_{\nu}^{\text {rec }}-E_{\nu}^{\text {true }}\right) / E_{\nu}^{\text {true }}$. 

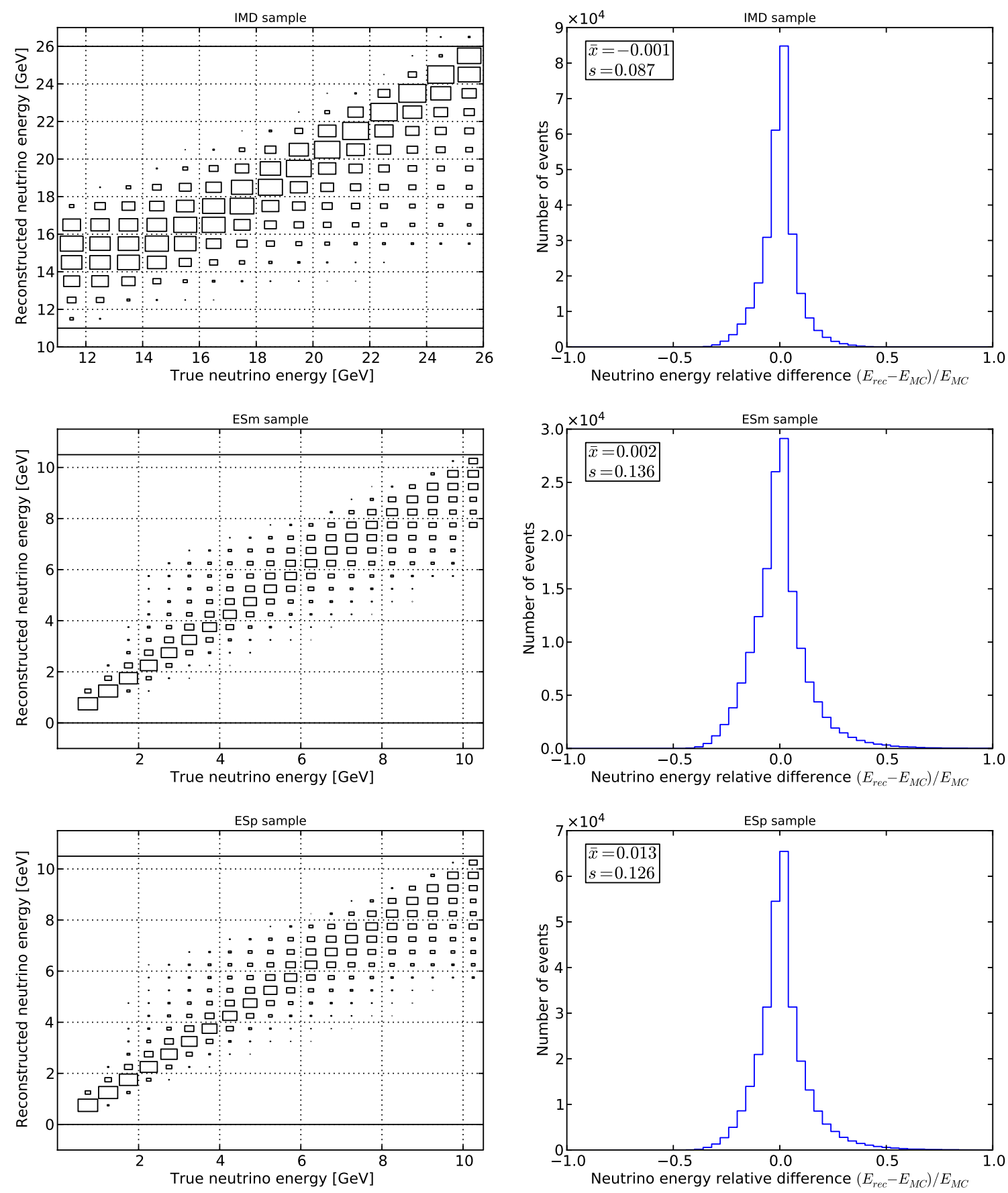

FIG. 15. Improved reconstruction of the neutrino energy. Left-migration matrix (the sum over each column is normalized to unity). The overflow and underflow bins are separated with horizontal lines. Right-relative difference $\left(E_{\nu}^{\text {rec }}-E_{\nu}^{\text {true }}\right) / E_{\nu}^{\text {true }}$ for the respective leptonic event sample (background excluded). Top—IMD sample; middle—ES ${ }^{-}$sample (10 GeV NF); bottom—ES ${ }^{+}$sample $(10 \mathrm{GeV}$ $\mathrm{NF})$.

\section{ESTIMATION OF THE NEUTRINO FLUX ENERGY DISTRIBUTION}

We have demonstrated that with the near detector we can measure neutrino-electron interaction rates and, in addition, we can estimate the neutrino energy on an event-byevent basis. However, there are two components with different flavor $\left(\nu_{\mu}\right.$ and $\bar{\nu}_{\mathrm{e}}$ for the $\mu^{-}$beam or $\bar{\nu}_{\mu}$ and $\nu_{\mathrm{e}}$ for the $\mu^{+}$beam) that contribute to each event sample (IMD, $\mathrm{ES}^{-}, \mathrm{ES}^{+}$). The $25 \mathrm{GeV}$ neutrino factory provides a unique opportunity in its $\mu^{-}$running mode to extract directly both flux components above the IMD threshold, because in this region events from two independent samples (IMD and $\mathrm{ES}^{-}$) are available. In all other cases, including the $10 \mathrm{GeV}$ neutrino factory, one needs to disentangle the two 

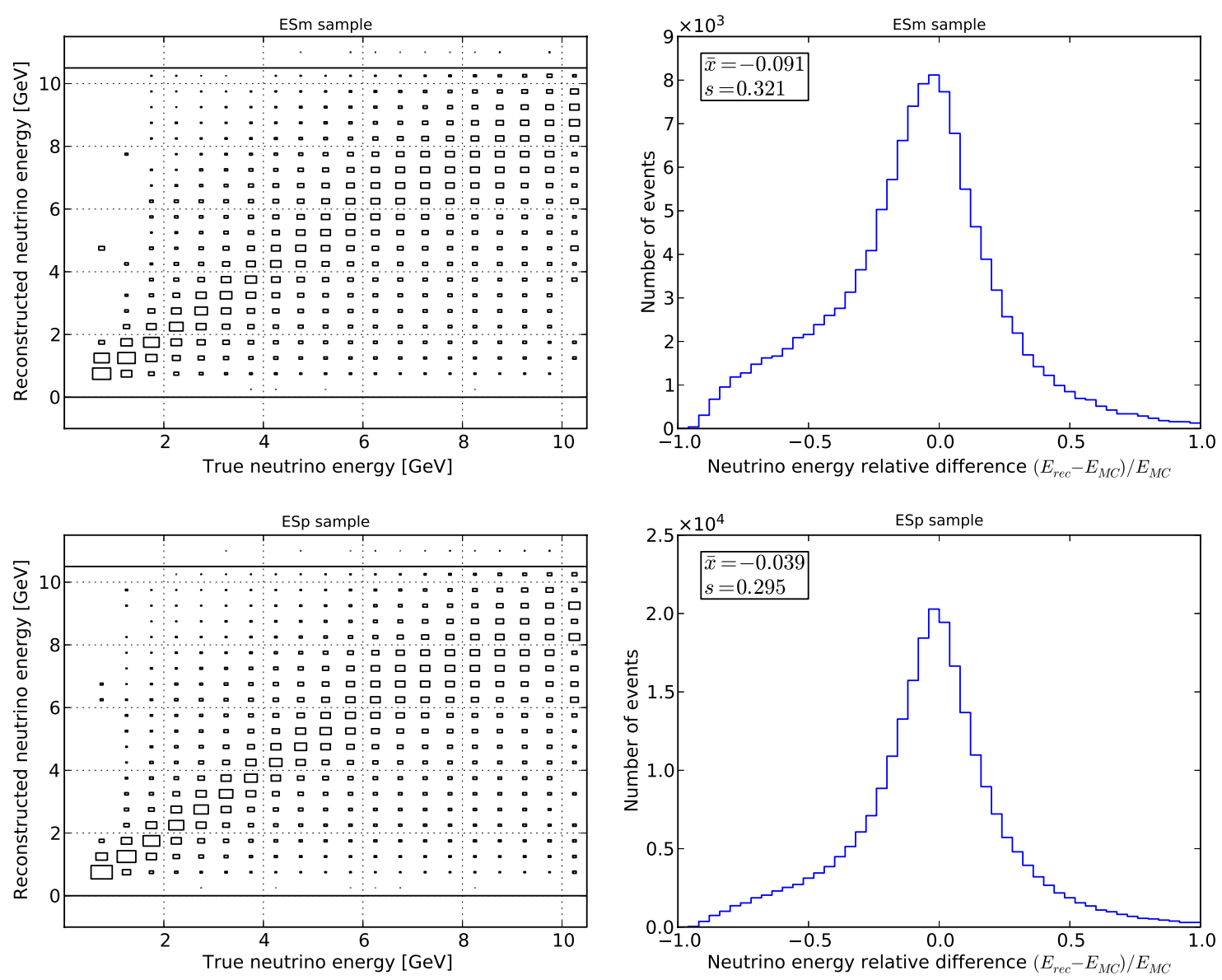

FIG. 16. Reconstruction of the neutrino energy using measured quantities. Left: Migration matrix (the sum over each column is normalized to unity). The overflow and underflow bins are separated with horizontal lines. Right: Relative difference $\left(E_{\nu}^{\text {meas }}-E_{\nu}^{\text {true }}\right) / E_{\nu}^{\text {true }}$ for the respective leptonic event sample (background excluded) at the $10 \mathrm{GeV}$ neutrino factory. Top-ES ${ }^{-}$sample; bottom—ES ${ }^{+}$sample.

components using a fitting procedure and/or Monte Carlo shape assumptions. For example, at a $10 \mathrm{GeV}$ neutrino factory and in $\mu^{-}$running mode one measures

$$
\begin{aligned}
N_{\mathrm{ES}^{-}}(E) & =N_{\nu_{\mu} \mathrm{e}^{-}}(E)+N_{\bar{\nu}_{\mathrm{e}} \mathrm{e}^{-}}(E) \\
& =\phi_{\nu_{\mu}}(E) \sigma_{\nu_{\mu}}^{N C} \mathrm{e}^{-}(E)+\phi_{\bar{\nu}_{\mathrm{e}}}(E) \sigma_{\bar{\nu}_{\mathrm{e}}}^{N C} \mathrm{e}^{-}(E),
\end{aligned}
$$

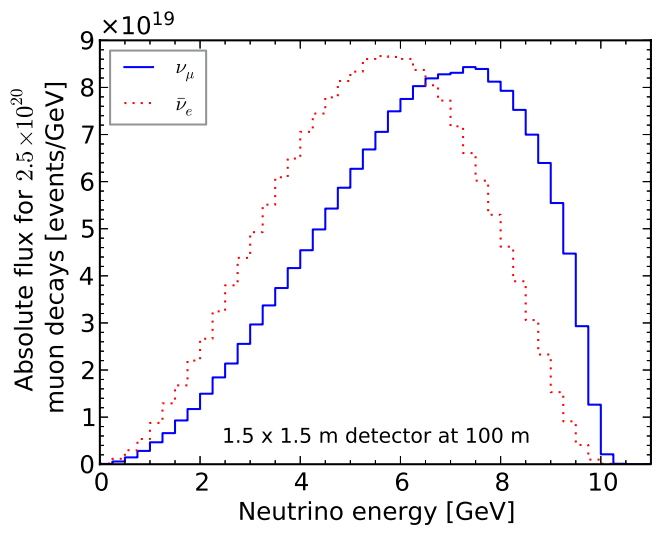

FIG. 17. Energy distributions of the $\nu_{\mu}$ and $\bar{\nu}_{\mathrm{e}}$ coming from $10 \mathrm{GeV}$ neutrino factory decay ring in $\mu^{-}$mode. where $N(E)$ denotes event rates, $\phi(E)$ beam fluxes, and $\sigma(E)$ cross sections. To resolve the fluxes in this case, one can make use of the different shapes of the electron and muon neutrino spectra, see Fig. 17. Moreover, neutrinos from both flavors are produced by the decays of muons from the same beam. Therefore, a strong correlation exists between the two spectral shapes as seen by the near detector. Of course, the extent of validity of a fitting procedure exploiting such correlation needs to be verified by respective Monte Carlo simulations.

\section{CHARM AND TAU DETECTOR}

The near detector at the neutrino factory needs to measure the charm cross section to validate the size of the charm background in the far detector, since this is one of the main backgrounds to the wrong-sign muon signature. The charm cross section and branching fractions are poorly known, especially close to threshold. The theoretical uncertainty in the charm cross section arises from the errors in the strange sea content of the nucleon, the semileptonic charm to muon branching fraction (with a $10 \%$ relative error), the longitudinal structure function $\left(F_{L}\right)$, and 
higher-twist effects. For this reason, it is paramount to make an independent near detector measurement of the charm cross section and make the error in the charm cross section negligible in the estimation of the neutrino oscillation background.

Since events with a $\tau$ lepton in the final state have a similar signature to charm events, any detector that can measure charm should be able to measure $\tau$ 's as well. This is important to explore couplings of nonstandard interactions (NSI) at source $\epsilon_{\tau \mu}^{s}, \epsilon_{\tau e}^{s}$ or detection $\epsilon_{\tau \mu}^{d}, \epsilon_{\tau e}^{d}$ (see Sec. 1.6 in [1] for a comprehensive treatment). A semiconductor vertex detector for charm and $\tau$-lepton detection could potentially be used for this purpose. The advantage of this type of detector is that it is able to operate at a high event rate and still have very good spatial resolution. This is necessary to distinguish the primary neutrino interaction vertex from the secondary vertex due to the short lived charm hadron or the $\tau$ lepton. The vertex detector could be similar to the NOMAD-STAR detector [18] that was installed upstream of the first drift chamber of the NOMAD neutrino oscillation experiment [19] used to measure the impact parameter and double vertex resolution to determine the charm detection efficiency. The reconstruction of $\tau$ leptons from an impact parameter signature with a dedicated silicon vertex detector was studied in the NAUSICAA proposal [20]. A silicon vertex detector with a $\mathrm{B}_{4} \mathrm{C}$ target was proposed as an ideal medium to identify $\tau$ leptons. Standard $\nu_{\mu} \mathrm{CC}$ interactions have an impact parameter rms of $28 \mu \mathrm{m}$, while tau decays have an impact parameter rms of $62 \mu \mathrm{m}$. By performing a cut on the impact parameter significance $\left(\sigma_{\mathrm{IP}} / \mathrm{IP}\right)$, one can separate one prong decays of the tau from the background. For three prong decays of the tau, a double vertex signature is used to separate signal from background. The total net efficiency of the tau signal in NAUSICAA was found to be $12 \%$.

A silicon strip vertex detector as part of the near detector could consist of a target of ten modules of $\mathrm{B}_{4} \mathrm{C}$ (this material has the largest density $2.49 \mathrm{~g} / \mathrm{cm}^{3}$ for the longest radiation length $21.7 \mathrm{~cm}$ ) of dimensions $100 \times 100 \times$ $2 \mathrm{~cm}^{3}$ per module for a total mass of $498 \mathrm{~kg}$ (Fig. 18). There are 40 ladders of silicon detectors, of $50 \mathrm{~cm}$ length, in each plane and there are 12 silicon planes in each detector. This implies 480 ladders, with nine silicon detectors per ladder, for a total of 4320 silicon detectors, covering a total area of $12 \mathrm{~m}^{2}$. Assuming 640 channels per ladder, it is a total of 307200 readout channels.

At a $25 \mathrm{GeV}$ neutrino factory, about $7 \times 10^{7} \nu_{\mu} \mathrm{CC}$ interactions per year are expected in a $500 \mathrm{~kg}$ detector. Assuming that the charm branching ratio is about $4 \%$ of the $\nu_{\mu} \mathrm{CC}$ rate at an average energy of $15 \mathrm{GeV}$ and with a similar detection efficiency to the NOMAD-STAR detector [18], one expects $2 \times 10^{5}$ charm events per year. A search for tau events in this detector from nonstandard interactions can also be carried out. With the tau detection efficiency found in NAUSICAA and a ratio of cross sections

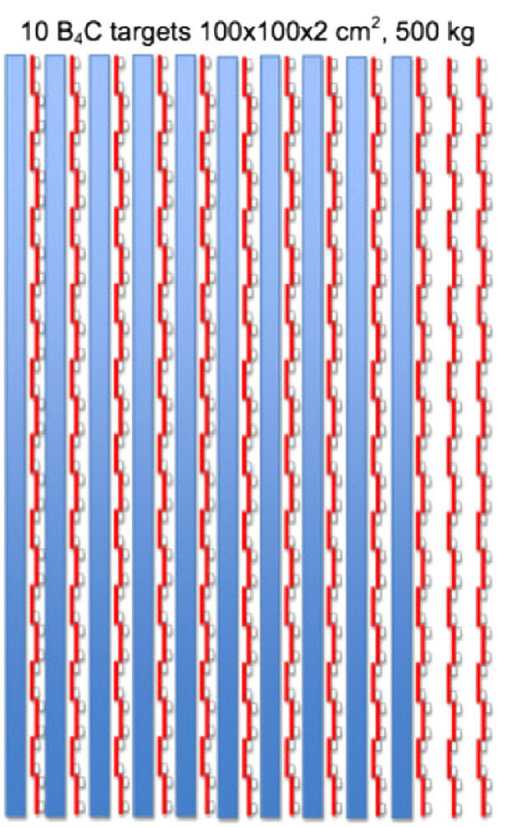

FIG. 18. Conceptual design for a vertex detector at a near detector of a neutrino factory.

$\sigma_{\tau} / \sigma_{\mu} \sim 0.4$ (at an average neutrino energy of $15 \mathrm{GeV}$ ) a $\mu-\tau$ conversion probability sensitivity of $P_{\mu \tau}<7 \times$ $10^{-8}$ at $90 \%$ C.L. is obtained for a 10 year run, assuming no background. This improves the current limits from NOMAD and CHORUS by 3 orders of magnitude [21,22].

At a $10 \mathrm{GeV}$ neutrino factory, however, the charm branching fraction is reduced to about $2 \%$ and the $\nu_{\mu} \mathrm{CC}$ interaction rate per year is also reduced to $1.3 \times 10^{7}$ in a $500 \mathrm{~kg}$ detector. Therefore, the yearly charm rate is $\sim 2 \times 10^{4}$ and the sensitivity to a $\mu-\tau$ conversion probability is also worse. Assuming a $\sigma_{\tau} / \sigma_{\mu} \sim 0.2$ at the lower energy, we obtain that $P_{\mu \tau}<7 \times 10^{-7}$ at $90 \%$ C.L. for a 10 year run.

\section{NEAR TO FAR DETECTOR EXTRAPOLATION}

A method to extrapolation flux results from the near detector to a far detector for neutrino oscillation searches was developed in [1]. The solid angle subtended by the near detector at the end of the decay straight of the neutrino factory is much larger than the solid angle observed by the far detector. This results in a different energy spectrum for a near detector compared to a far detector. A robust method for predicting the far detector flux, based on the near detector flux, was developed in the context of a neutrino factory.

The technique essentially involves three matrices describing the setup: near detector response, flux projection and far detector response; in addition to cross-section matrices for the relevant processes and a parametrization of the oscillation probability which is ultimately used in the determination of the sensitivity to oscillation parameters. The method relies on a fit to the observed far 

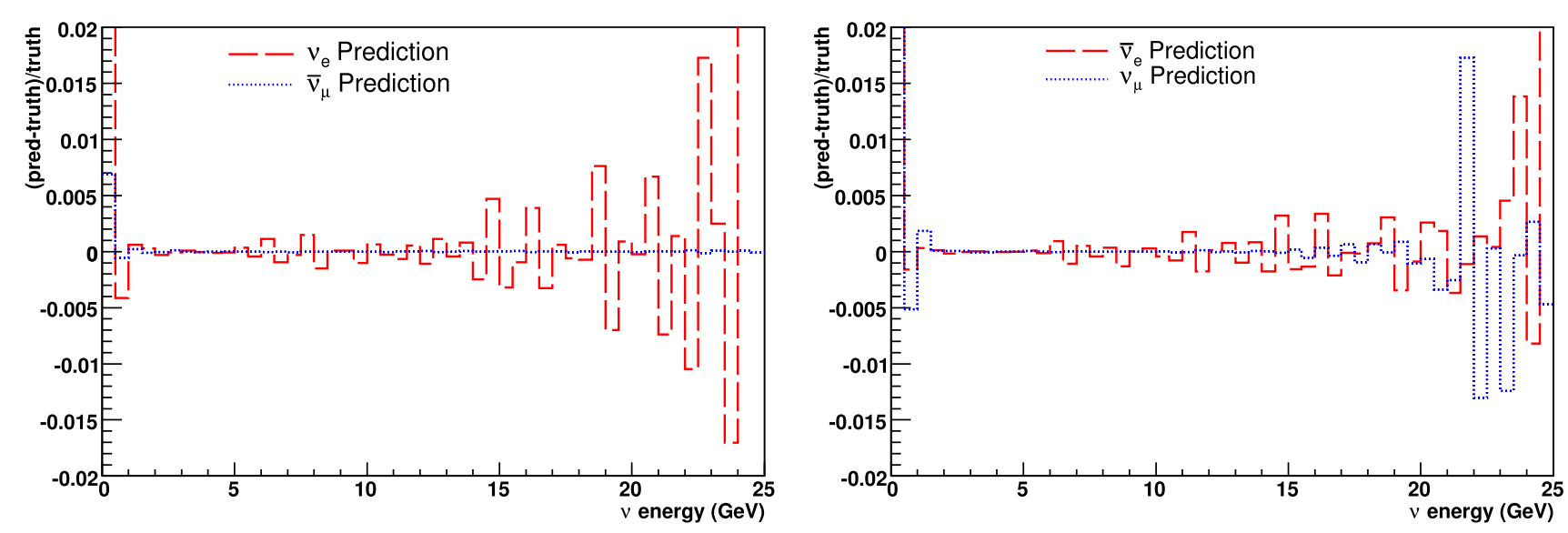

FIG. 19. Comparison of predicted to true flux through the ND for stored $\mu^{+}$(left) and stored $\mu^{-}$(right).

detector spectrum directly by using the projection of the observed near detector spectrum [23]. That is, the predicted spectrum for a given grid point on the $\left(\theta_{13}, \delta\right)$ plane is calculated as

$$
N_{\mathrm{FD}}=M_{\mathrm{FD}} P_{\mathrm{osc}}\left(\theta_{13}, \delta\right) M_{n O s c} M_{N D}^{-1} N_{N D},
$$

where $N_{F D}$ and $N_{N D}$ are the observed far and near detector spectra respectively, $M_{F D}$ and $M_{N D}$ are matrices representing the combination of cross section and response for $\nu_{\mu}\left(\bar{\nu}_{\mu}\right)$ at the far detector and $\nu_{e}\left(\bar{\nu}_{e}\right)$ at the near detector respectively, $P_{\text {osc }}$ is the oscillation probability and $M_{n O s c}$ relates the expected far detector $\nu_{e}\left(\bar{\nu}_{e}\right)$ flux without oscillations to the expected $\nu_{e}\left(\bar{\nu}_{e}\right)$ flux at the near detector. The extracted function is then fit to the oscillation probability formulas to find the best fit values of the $\theta_{13}$ and $\delta$ simultaneously.

Using the near detector to measure both the $\nu_{\mu}\left(\bar{\nu}_{\mu}\right)$ and $\bar{\nu}_{e}\left(\nu_{e}\right)$ rates, the interaction spectra at the far detector can then be predicted. The only background to the $\nu_{\mu}\left(\bar{\nu}_{\mu}\right)$ measurement is likely to be from neutral current interactions. Using a combination of missing $p_{T}$ and vertex reconstruction, both of which can be measured with high resolution at a near detector, this could be suppressed to at least the level in the far detector. The $\bar{\nu}_{e}\left(\nu_{e}\right)$ measurement can be carried out with electron scattering events, as shown above. Projection of the predicted flux is carried out using scaling matrices calculated using a comparison of the true fluxes at the near and far sites. The near detector resolution is used to construct a probability matrix relating directly near detector interactions to unoscillated far detector interactions, as in MINOS [24].

An initial study of the power of this technique was carried out assuming a $100 \mathrm{~kg}$ cylindrical detector of $1 \mathrm{~m}$ radius placed $100 \mathrm{~m}$ from the end of a $600 \mathrm{~m}$ straight decay section at a $25 \mathrm{GeV}$ neutrino factory. The flux expected at the near detector site is predicted by randomly generating muon decays along a straight line with an appropriate beam divergence and calculating the expected spectrum from the detector acceptance as calculated for each decay position. The detector is modeled using a conservative estimate of the $\bar{\nu}_{e}\left(\nu_{e}\right)$ energy resolution of $35 \% / \sqrt{E}(\mathrm{GeV})$ with efficiency rising linearly from $0 \%$ at $0 \mathrm{GeV}$ up to $70 \%$ above $4 \mathrm{GeV}$. The $\nu_{\mu}\left(\bar{\nu}_{\mu}\right)$ resolution is set at $20 \% / \sqrt{E}(\mathrm{GeV})$, with efficiency of $80 \%$ for $\bar{\nu}_{\mu}$ and $60 \%$ for $\nu_{\mu}$ above $4 \mathrm{GeV}$ (similar to the far detector).

A smear is performed on the calculated interactions at the near detector and the flux and correlation matrices for each channel are then projected to the appropriate far detector in bins of width $0.5 \mathrm{GeV}$. A comparison of the near and far detector fluxes is shown in Fig. 19). Differences of more than $1 \%$ with respect to the predicted fluxes are only visible at the highest energies.

The far detector spectra are calculated with the nonoscillation predictions from the near detector. The far detector spectra obtained are then used to perform a fit using the function

$$
\chi^{2}=\sum_{i} \sum_{j}\left(N_{i, j}-n_{i, j}\right) V_{i, j}^{-1}\left(N_{i, j}-n_{i, j}\right)^{T},
$$

where $i$ is the detector baseline, $j$ the polarity, $N$ the predicted spectrum, $n$ the data spectrum, and $V$ the correlation matrix, composed of the projected matrix of the prediction and the expected errors on the far detector measurement. Figure 20 shows the results of fits to a range of $\theta_{13}$ and $\delta$ values using this technique. The resolution of $\theta_{13}$ and $\delta$ is generally better at large $\theta_{13}$.

A comparison of $\chi^{2}$ fits obtained by performing the near-far extrapolation method compared to a method in which the neutrino flux is part of the fit without being constrained by near detector data are shown in Fig. 21, for the true values of $\theta_{13}=1^{\circ}$ and $\delta=45^{\circ}$. At the $1 \sigma$ level $\left(\chi^{2}-\chi_{\min }^{2}=1\right)$ the fits to $\theta_{13}$ and $\delta$ are very similar for both methods. However, the near-far projection method shows smaller projected errors in both $\theta_{13}$ and $\delta$ at the $3 \sigma$ level $\left(\chi^{2}-\chi_{\min }^{2}=9\right)$. 


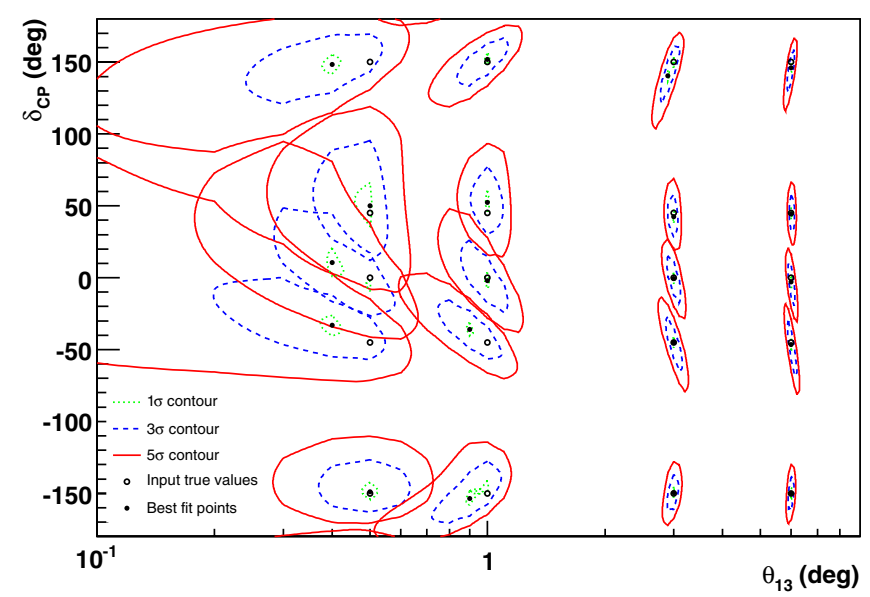

FIG. 20. Fits to simulated data using the near-far prediction assuming a normal mass hierarchy.

Figure 22 shows the trend for the measurement of the oscillation parameters for the near-far projection method. The typical $1 \sigma$ error expected for $\theta_{13}$ is $\Delta \theta_{13} \sim 0.05^{\circ}$. The error in $\delta$ depends on the value of $\delta$ and at larger values of $\theta_{13}$ is $\Delta \delta \sim 5^{\circ}$. Both $\theta_{13}$ and $\delta$ are predicted by the fit without any significant bias.

\section{CONCLUSIONS}

We have proposed a conceptual design for the tracker part of the neutrino factory near detector. A full Monte Carlo simulation of neutrino interactions and particle transport was implemented using standard tools. An idealized detector geometry and simplified signal digitization was used in the MC simulation. Basic reconstruction algorithms were developed to extract information relevant for neutrino-electron scattering measurements. Selection procedures, which increase the signal-to-background ratio from $\sim 10^{-4}$ initially to $\sim 10$, are defined. A onedimensional signal extraction analysis was performed. It is demonstrated that the number of neutrino-electron scattering events can be measured from the $\theta_{\ell}^{2} E_{\ell}$ variable histogram. A direct comparison between measured and true number of signal events shows a deviation of $1 \%-2 \%$, which is also consistent with expected statistical fluctuation from the simulated sample size. In the case of a $10 \mathrm{GeV}$

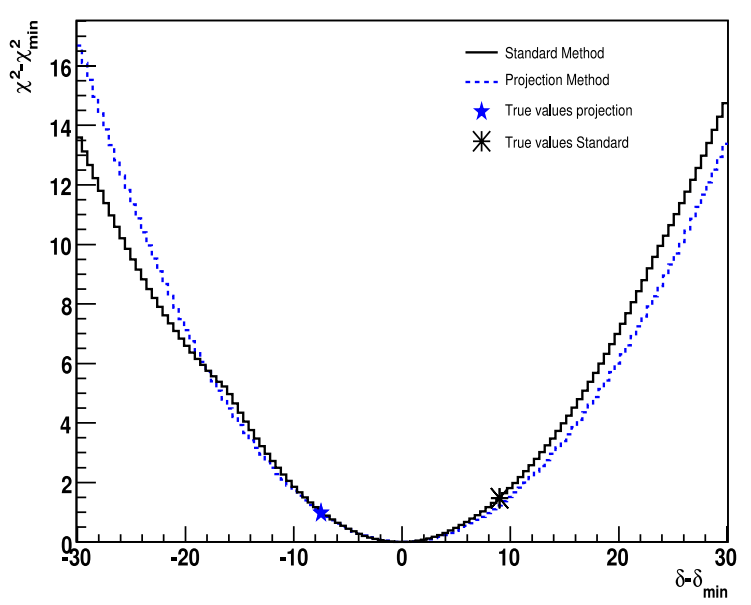

FIG. 21. Projection of the minimum $\chi^{2}$ onto the $\theta_{13}$ axis (left) and the $\delta$ axis (right) for $\theta_{13}=1^{\circ}$ and $\delta=45^{\circ}$.
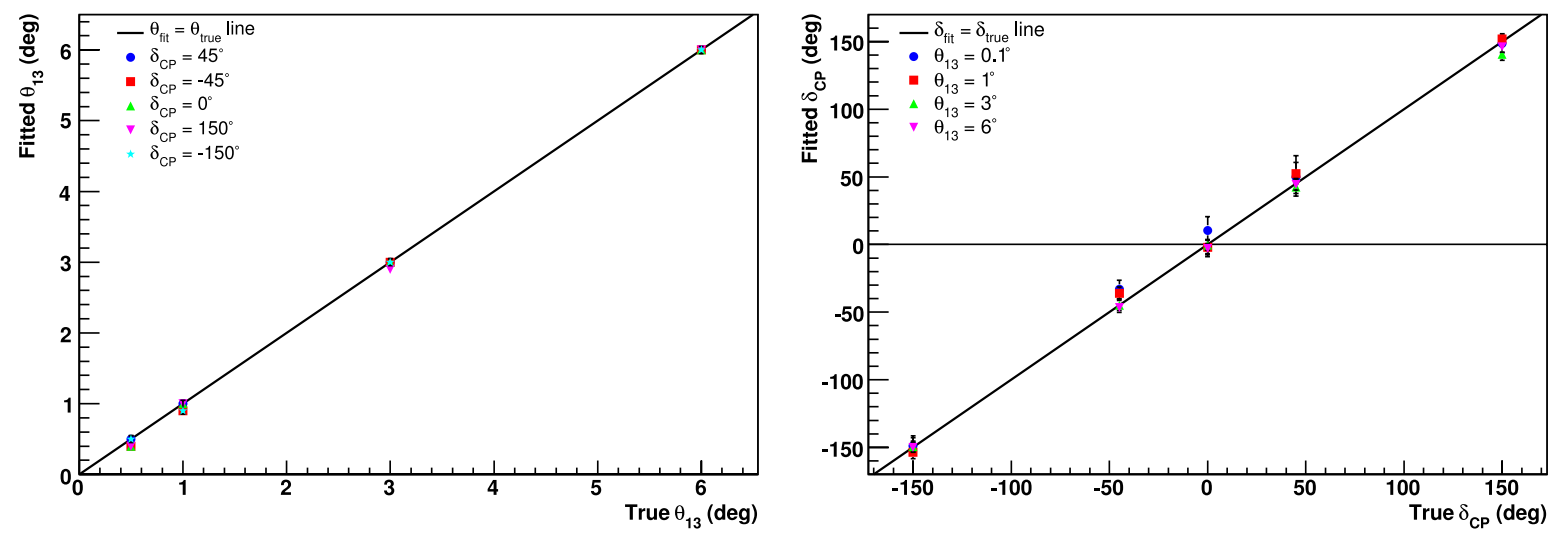

FIG. 22. Quality of fit to $\theta_{13}$ (left) and $\delta$ (right) for a range of values, using the near-far projection method. 
neutrino factory, with the performance presented herein and assuming $5 \times 10^{20}$ muon decays per mode per year, an independent absolute normalization of both running modes to $1 \%$ can be achieved within one year of nominal operation. With the achievable signal-to-background ratio of $\sim 10$, one needs to control the systematics of the background estimation to $10 \%$ to match the statistical uncertainty. It is worth noting that, apart from assumptions of muon identification and calorimetric measurement of EM energy, MC truth was not used in the reconstruction and signal extraction. Therefore, the results obtained are a reliable approximation to results obtainable at a real experiment, provided that the MC simulation is close to reality.

A design for a silicon vertex detector upstream of the tracker was shown to deliver about $2 \times 10^{5}$ charm events per year at a $25 \mathrm{GeV}$ neutrino factory and $2 \times 10^{4}$ at a $10 \mathrm{GeV}$ neutrino factory near detector. Additionally, it will be able to search for nonstandard interactions by showing that it can achieve a $\mu-\tau$ conversion probability sensitivity of $P_{\mu \tau}<7 \times 10^{-8}$ at $90 \%$ C.L. for a 10 year run at a $25 \mathrm{GeV}$ neutrino factory and $P_{\mu \tau}<7 \times 10^{-7}$ for the same period at a $10 \mathrm{GeV}$ neutrino factory.

The near and far detectors at a neutrino factory cover very different solid angles with different neutrino energy distributions. Despite this, an extrapolation method from the near to the far detector was developed in which the values of $\theta_{13}$ and $\delta$ are predicted by the fit without any significant bias and the errors in both quantities are expected to be $\Delta \theta_{13} \sim 0.05^{\circ}$ and $\Delta \delta \sim 5^{\circ}$.

\section{ACKNOWLEDGMENTS}

We acknowledge the financial support of the European Community under the European Commission Framework Programme 7 Design Study: EUROnu, Project No. 212372, as well as the co-financing by the National Science Fund of Bulgaria under Contract No. DO 02-149/ 07.10.2009 and the Science and Technology Facilities Council (STFC) from the UK.

[1] R. J. Abrams et al., arXiv:1112.2853.

[2] A. Laing, Ph.D. thesis, University of Glasgow, 2010.

[3] S. R. Mishra, R. Petti, and C. Rosenfeld, Proc. Sci., NUFACT08 (2008) 069 [arXiv:0812.4527].
[4] L. B. Okun, Leptons and Quarks (Nauka, Moscow, 1990), 2nd ed.

[5] Y. Karadzhov, AIP Conf. Proc. 1222, 467 (2010) [http:// link.aip.org/link/?APCPCS/1222/467/1].

[6] Y. Karadzhov and R. Tsenov, Annual of the University of Sofia, Faculty of Physics (2010), Vol. 103 [http://phys.unisofia.bg/annual/arch/103/arch_en.html].

[7] C. Andreopoulos et al., Nucl. Instrum. Methods Phys. Res., Sect. A 614, 87 (2010).

[8] S. Agostinelli et al. (GEANT4 Collaboration), Nucl. Instrum. Methods Phys. Res., Sect. A 506, 250 (2003).

[9] A possible realization of such a calorimeter could follow the design of the EM calorimeter of the HiResM $\nu$ detector [3] proposed to serve as near detector in the LBNE experiment at Fermilab. The energy resolution mentioned above is obtained by MC simulation.

[10] A. Cervera-Villanueva, J. J. Gómez-Cadenas, and J. A. Hernando, Nucl. Instrum. Methods Phys. Res., Sect. A 534, 180 (2004).

[11] R. E. Kalman, J. Basic Eng. 82, 35 (1960).

[12] R.E. Kalman and R. Bucy, J. Basic Eng. 83, 95 (1961).

[13] P. Billoir, R. Fruhwirth, and M. Regler, Nucl. Instrum. Methods Phys. Res., Sect. A 241, 115 (1985).

[14] The analysis applied for the $25 \mathrm{GeV}$ neutrino factory scenario did not assume muon/electron identification and did not use anticipated calorimetric measurement of the electron energy. This results in a somewhat poorer background rejection.

[15] Direct comparison of signal-to-background ratios and distributions from 25 and $10 \mathrm{GeV}$ scenarios could be misleading because of the additional assumptions and background cuts applied to the former.

[16] D. Geiregat et al. (CHARM-II Collaboration), Phys. Lett. B 247, 131 (1990).

[17] P. Vilain et al. (CHARM-II Collaboration), Phys. Lett. B 364, 121 (1995).

[18] M. Ellis and F. J. P. Soler, J. Phys. G 29, 1975 (2003).

[19] J. Altegoer et al. (NOMAD Collaboration), Nucl. Instrum. Methods Phys. Res., Sect. A 404, 96 (1998).

[20] J. J. Gomez-Cadenas, J. A. Hernando, and A. Bueno, Nucl. Instrum. Methods Phys. Res., Sect. A 378, 196 (1996).

[21] P. Astier et al. (NOMAD Collaboration), Nucl. Phys. B 611, 3 (2001).

[22] E. Eskut et al. (CHORUS Collaboration), Nucl. Phys. B 793, 326 (2008).

[23] A. Laing and F. J.P. Soler, Proc. Sci., NUFACT08 (2008) 129.

[24] D. G. Michael et al. (MINOS Collaboration), Phys. Rev. Lett. 97, 191801 (2006). 